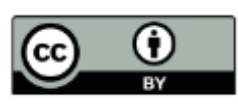

\title{
Salud y privación farmacéutica en España. Una devaluación de la ciudadanía social*
}

\author{
Health and pharmaceutical deprivation in \\ Spain. A devaluation of social citizenship
}

\section{Saúde e privação farmacêutica em Espanha. \\ Uma desvalorização da cidadania social}

Recibido: 18 de Agosto de 2019. Aceptado: 10 de

Febrero de 2020. Publicado: 1 Octubre de 2020.

DOI: https://doi.org/10.11144/Javeriana.rgps19.spfe

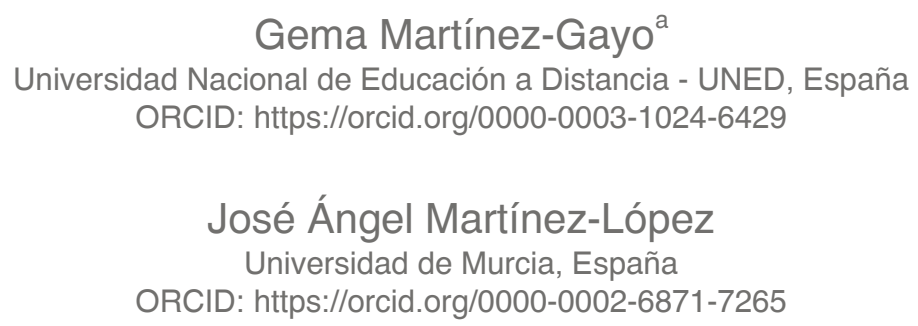

Para citar este artículo Martínez-Gayo G, Martínez-López JA. Salud y privación farmacéutica en España. Una devaluación de la ciudadanía social. Rev Gerenc Polit Salud. 2020;19. https://doi.org/10.11144/Javeriana.rgps19 .spfe

a Autora de correspondencia. E-mail: gmartinez9@alumno.uned.es 


\section{Resumen}

El acceso a la salud es uno de los pilares básicos del estado de bienestar español. Desde finales de los años setenta, los cambios legislativos introducidos han modificado tanto las condiciones de acceso como el grado de cobertura estatal de los productos farmacéuticos. El sistema de copago farmacéutico establecido en España ha sufrido cambios sustanciales recientes, tanto en la cobertura de medicamentos como en el aporte económico para acceder a estos. El objetivo de esta investigación es conocer las implicaciones de dichas reformas en la población y determinar cuáles son los perfiles más afectados. La aproximación al objeto de estudio se ha realizado siguiendo un pluralismo metodológico. Se analizaron los principales cambios normativos y las fuentes de datos relacionadas con el acceso a productos farmacéuticos. Los resultados confirman la falta de progresividad de las reformas legales en relación al copago sanitario. Igualmente, se muestra un incremento de las dificultades de acceso a los medicamentos prescritos por parte de las personas más vulnerables, en este caso, las mujeres y los hogares con menor renta disponible.

Palabras clave: Pobreza, fármacos, salud, desigualdad social, legislación sanitaria.

\section{Resumo}

O acesso à saúde é um dos pilares básicos do estado de bem-estar espanhol. Desde finais dos anos setenta, as mudanças legislativas introduzidas têm transformado tanto as condições de acesso quanto o grau de cobertura estatal dos produtos farmacêuticos. O sistema de co-pagamento farmacêutico estabelecido em Espanha tem sofrido mudanças substanciais recentes, tanto na cobertura de medicamentos como no aporte econômico para aceder aos mesmos. O objetivo desta pesquisa é conhecer as implicações de tais reformas na população e determinar quais os perfis mais afetados. A aproximação ao objeto de estudo se tem realizado seguindo um pluralismo metodológico. Analisaram-se as principais mudanças normativas e as fontes de dados relacionados com o acesso a produtos farmacêuticos. Os resultados confirmam a falta de progressividade das reformas legais em relação ao co-pagamento sanitário. Igualmente, mostrase um incremento das dificuldades de acesso aos medicamentos prescritos por parte das pessoas mais vulneráveis, neste caso, as mulheres e os lares com menor renda disponível.

Palavras-chave: Pobreza, fármacos, saúde, desigualdade social, legislação sanitária.

\section{Abstract}

Access to health is one of the basic pillars of the Spanish welfare state. Since the late 1970s, legislative changes introduced have modified both access conditions and the degree of state coverage of pharmaceutical products. Since the late 1970s, legislative changes have been introduced that have modified both the conditions of access and the degree of state coverage of pharmaceutical products. The pharmaceutical co-payment system established in Spain has undergone recent substantial changes in both drug coverage and the economic contribution to access them. This research aims to know the implications of these reforms in the population and determine the most affected profiles. The approach to the object of study has been made following a methodological pluralism. Main legislative changes and data sources related to the access to pharmaceutical products were analyzed. Results confirm the lack of progressivity of the legal reforms concerning the health co-payment. Furthermore, they show an increase in the difficulties of access to prescribed medicines for the most vulnerable people, in this case, women and those households with lower income.

Keywords: Poverty, drugs, health, social inequality, health legislation. 


\section{Introducción}

Uno de los rasgos identificativos del Estado de bienestar es la protección social en materia de salud. Ante los infortunios que pueda sufrir una persona, éste protege a la ciudadanía democratizando el derecho a la salud en sus dos modalidades principales: la atención sanitaria directa -ya sea primaria, especializada u hospitalaria-, y la atención farmacéutica.

Con la instauración de la democracia en España, tras el fin del régimen dictatorial a finales de la década de 1970, los gobiernos progresistas comenzaron a desarrollar un sistema de salud universalista. Éste ha sido un referente dentro del contexto europeo ya que no está ligado a las contribuciones derivadas del empleo sino al concepto de ciudadano, constituyendo una expresión de la ciudadanía social. El Estado de bienestar en España se fue desarrollando abarcando cada vez nuevos retos más allá de la Sanidad, Educación y Seguridad Social.

Con la llegada del nuevo milenio y la introducción del euro, España empezó a perder autonomía para la inversión en nuevas políticas públicas dadas las necesidades de adecuarse a las exigencias de la autoridad fiscal europea. Posteriormente, la crisis económica llevó consigo el desarrollo de políticas neoliberales orientadas a salvaguardar las balanzas fiscales de la mayoría de los países de la zona euro en detrimento del desarrollo de políticas sociales para proteger a la población ante los nuevos retos: el aumento del desempleo, reducción de los salarios, aumento de la pobreza y desigualdad social, restricción en el acceso de derechos sociales (Sanidad, Educación y Seguridad Social, principalmente). El acceso a la prestación farmacéutica ha sido uno de los que se ha visto más afectados como consecuencia del aumento del copago farmacéutico y la exclusión de determinados medicamentos -especialmente los relacionados con enfermedades crónicas- del catálogo de fármacos estatal.

En este contexto se busca determinar cuáles son las implicaciones en la población de las restricciones en materia de cobertura de medicamentos y si existe un perfil determinado asociado a este tipo de privación. Para ello, se hizo una revisión teórica de los aportes más destacados en este campo, a la vez que se estudió la evolución de las medidas legislativas que ha implementado el gobierno español en este ámbito. Posteriormente, se desarrolla un análisis de datos secundarios sobre gasto farmacéutico y dificultades de acceso a dichos productos en los últimos años.

Este trabajo es una primera aproximación a un fenómeno escasamente abordado en España. Sin embargo, se considera que tiene importantes repercusiones en la protección social, en las políticas sociales y la configuración del Estado de bienestar. Se trata de un planteamiento novedoso ya que vislumbra resultados de un nuevo tipo de privación material que incide directamente en la calidad de vida y salud de la población. 


\section{Protección a la salud en España: un sistema en permanente cambio}

La protección a la salud en España aparece plasmada en la Constitución Española de 1978 en su artículo 43, según el cual:

Se reconoce el derecho a la protección de la salud. Compete a los poderes públicos organizar y tutelar la salud pública a través de medidas preventivas y de las prestaciones y servicios necesarios (art. 43 Constitución Española de 1978).

Por tanto, se contempla la responsabilidad en la gestión de este bien, incluyendo los servicios y prestaciones necesarias para la garantizar la atención de la salud.

En 1986 se aprueba la Ley 14/1986 General de Sanidad, como marco para hacer efectivo el artículo 43 de la Constitución Española, especificando en su artículo 107 que el gasto farmacéutico está comprendido tanto por la suma del gasto en productos farmacéuticos y sanitarios -derivado de la expedición de la receta oficial u orden de dispensación del Sistema Nacional de Salud en oficinas de farmacia-, como por el gasto farmacéutico hospitalario del Sistema Nacional de Salud.

La estructura del sistema sanitario español está descentralizada desde el año 2001 cuando se aprobó la Ley 21/2001, transfiriendo las competencias en materia sanitaria a las Comunidades Autónomas. Este ha sido el cambio más importante en el sistema sanitario español desde que en 1986 se aprobó la Ley General de Sanidad. Posteriormente, en 2011 se produjo una importante transformación en la gestión de los productos farmacéuticos cuando se aprobó la prescripción de los medicamentos según el principio activo y no marcas comerciales, suponiendo un ahorro para las arcas públicas.

Los mecanismos de financiación del sistema sanitario español son muy diferentes a los de la mayoría de los países europeos. Los ingresos provienen de dos vías fundamentalmente. En primer lugar, a través de los Presupuestos Generales del Estado y su posterior transferencia a las Comunidades Autónomas. En segundo lugar, a través de la financiación general que cada Comunidad Autónoma establece para tal fin pudiendo incluir el establecimiento de impuestos específicos. A modo de ejemplo, Cataluña estableció un impuesto de un euro por receta y otros territorios implantaron el llamado céntimo sanitario a los hidrocarburos. 


\section{Implicaciones de la privación farmacéutica en España}

\section{Los límites al derecho a la salud}

El acceso a la salud desde un plano universal implica socializar los riesgos que pueden tener las personas a lo largo de la vida garantizándose una cobertura sanitaria. Por tanto, supone un avance en ciudadanía social (1) ya que los ciudadanos tienen los mismos derechos en salud independientemente de su origen social. Esta protección social engloba tanto la asistencia médica -hospitalaria, primaria y especializada- como la atención farmacológica.

La atención sanitaria desde el planteamiento clásico salud/curación se encuentra superado en la actualidad y las corrientes actuales vinculan el concepto de salud al ámbito comunitario. Esta nueva perspectiva se engloba dentro de la nueva salud pública entendida como una síntesis de actuaciones preventivas y curativas, pero poniendo el enfoque tanto en las políticas públicas como en las actuaciones individuales y colectivas.

La industria farmacéutica se está viendo favorecida por un fenómeno global, que es más acuciante en las economías más desarrolladas: la medicalización y medicamentalización. La medicalización se caracteriza por el incremento de intervenciones médicas (diagnósticos y tratamientos) ante situaciones alejadas del concepto de enfermedad (2). La medicamentalización se diferencia de la medicalización porque atribuye a la profesión médica facultades con relación al monopolio y el control social de los individuos a través de los medicamentos (3). En todos estos conceptos hay un denominador común: la influencia de la industria farmacéutica en los procesos de salud/enfermedad desplazando, en parte, el poder de las instituciones sanitarias para regular dichos procesos.

Desde un plano internacional el acceso a los medicamentos es muy distinto atendiendo a la capacidad de renta y desarrollo científico de un país, así como el estatus de sus individuos. Páez (4) sostiene que: "Reducir las desigualdades en la salud de un individuo o comunidad tiene que ver con disminuir la brecha en el estatus actual de salud respecto al nivel óptimo" (4 p.204).

Este es un elemento clave para que parte de los individuos no perciban una restricción de su ciudadanía social en relación con el acceso de un bien de primera necesidad como son los medicamentos. En este sentido, la prevención de enfermedades y el desarrollo de hábitos saludables es una propuesta diferencial y alternativa al modelo clásico, donde las conductas individuales y colectivas son determinantes para el buen vivir (5).

La protección a la salud como derecho universal tiene un carácter social, y es considerado por numerosos autores como una responsabilidad del Estado para hacer efectiva la justicia social entre los ciudadanos desde un punto de vista equitativo, es decir, democrático. Rawls ha sido uno de los teóricos que ha profundizado sobre la idea de la justicia social. Su idea de justicia social está caracterizada por el hecho de que: 
Nadie sabe cuál es su lugar en la sociedad, su posición, clase o status social; nadie sabe tampoco cuál es su suerte en la distribución de ventajas y capacidades naturales, su inteligencia, su fortaleza, etc. (...) Los principios de la justicia se esconden tras el velo de la ignorancia (6 p.25).

En esta línea, "la justicia no impone restricciones respecto al tipo de desigualdades que son permisibles; únicamente exige que se mejore la posición de cada uno" (6 p.70). Por tanto, su idea de justicia es, en sí misma, una teoría sobre la justicia equitativa. En un escenario de destrucción de empleo y precarización de las condiciones de trabajo como el que ha tenido España en la última década, el empleo no es el elemento de protección que permita eludir las situaciones de desigualdad ya que se produce una igualdad desde la base, es decir, desde aquellos que se encuentran en una posición social inferior.

Por su parte, Sen (7) considera que en el análisis de la igualdad y la diversidad humana hay que atender a los factores personales incluyendo la propensión a la enfermedad, las condiciones físicas y mentales. Desde esta línea no podemos afirmar que la ciudadanía tenga las mismas posibilidades para acceder al mercado de trabajo y que la posición que ocupa cada uno y sus salarios sean el principal argumento para la diferenciación social. En sentido amplio, la privación de derechos restringe la ciudadanía social, no sólo en el ámbito sanitario sino también en cualquier aspecto relacionado con el bienestar individual. El desempleo, la precarización de las condiciones laborales, la pobreza extrema, las limitaciones e inseguridad con relación al acceso y el mantenimiento de la vivienda, etc., son ejemplos de situaciones que restringen el derecho al ejercicio efectivo de la ciudadanía social. En el caso concreto de la privación en el acceso de productos farmacéuticos $\mathrm{y}$, por tanto, a la salud, supone una manifestación contraria a la justicia social (6) y la libertad real de los ciudadanos, un obstáculo para alcanzar la ciudadanía social (1) y contraria a los principios sobre los que se constituyó el Estado de bienestar en los países europeos.

\section{Privación farmacéutica y pobreza: una nueva realidad social}

El acceso a una atención sanitaria es un derecho ampliamente aceptado en la comunidad internacional. De hecho, aparece recogido en el artículo 25 de la Carta de los Derechos Humanos aprobada por la ONU el 10 de diciembre de 1948:

Toda persona tiene derecho a un nivel de vida adecuado que le asegure, así como a su familia, la salud y el bienestar, y en especial la alimentación, el vestido, la vivienda, la asistencia médica y los servicios sociales necesarios (art. 25, ONU 1948).

Realmente no se hace referencia a un solo derecho sino a un conjunto que posibilita el bienestar de la ciudadanía. El Estado tiene un papel central a la hora de regular el acceso a los productos farmacéuticos donde participan tanto el mercado, a través de la industria farmacéutica, como el sistema público, garante de la asistencia a la misma. Por tanto, es corresponsable de la privación 
farmacéutica y como garante de la salud, tiene la posibilidad de establecer fórmulas para evitarla. Como sostiene Vargas-Chaves (8):

El problema del acceso a los medicamentos no obedece siempre al rol cuestionable de la industria farmacéutica, como sí en gran medida a los Estados, responsables inmediatos por el abandono de millones de pacientes en los países bajo el umbral de pobreza y desarrollo (8 p.168).

En España, la crisis económica favoreció el inicio de cambios legislativos que modificaron sustancialmente el acceso y el copago de productos farmacéuticos. Dichas modificaciones son consideradas por numerosos autores como un cambio en la configuración existente en el modelo de atención a la salud (9-11).

Esta situación es especialmente visible en el caso de las personas en situación de dependencia, las cuales suelen presentar adherencia a varios fármacos prescritos por sus facultativos. Un reciente estudio realizado por Monteverde et al. (12), en España, México y Argentina resalta que para establecer planes integrales y diagnosticar las necesidades de los colectivos con mayor vulnerabilidad es necesario:

Conocer los factores de riesgo más vinculados a la situación de dependencia en cada una de las poblaciones, las características socioeconómicas, de salud, de acceso a prestaciones médicas, de tipo de ayudas formales e informales para la realización de actividades de la vida diaria, así como las necesidades de ayudas insatisfechas (12 p.151).

Como consecuencia del progresivo envejecimiento de la población, la presión económica de la prestación farmacológica es cada vez mayor para quienes se encuentran en situación de dependencia. Estas personas precisan de una mayor atención sanitaria y farmacéutica, suponiendo la compra de productos farmacéuticos uno de los principales destinos de las prestaciones económicas destinadas a su cuidado en el ámbito familiar (13).

Por tanto, el acceso a la salud y a los medicamentos es uno de los grandes retos a los que se enfrentarán los países en los próximos años. Además, en el análisis del uso de los fármacos no es posible obviar el acceso y el desarrollo de los medicamentos genéricos. Estos productos farmacéuticos son más económicos pero existen importantes diferencias entre países. Como muestra el estudio realizado por Guttier et al. (14):

En los Estados Unidos y Alemania, por ejemplo, esta proporción es del $60 \%$ en volumen, mientras que en otros países, como España, Francia y Brasil, los medicamentos genéricos tienen entre un $27 \%$ y un $42 \%$ de cuota de mercado (14 p.2628).

La UE5 -de la que España es miembro junto a Alemania, Francia, Italia y Reino Unidoes el segundo consumidor de medicamentos a nivel mundial, tan sólo superado por EE.UU. (15). El consumo de medicamentos en España es muy elevado. Quizá podría considerarse que el envejecimiento de la población sea el factor determinante pero los datos sugieren la influencia de otros elementos. Por ejemplo, España es el séptimo país con mayor consumo de 
antidepresivos en 2017 (16) y es el tercer país con mayor consumo de antibióticos por cada 1000 habitantes (17). Por tanto, podemos observar la influencia de los fenómenos de la medicalización y medicamentalización en España, conductas alejadas de los determinantes sociales de la salud. Esta perspectiva es totalmente contraria a los principios fundamentales del Estado de bienestar español donde se prima no sólo la justicia social en el acceso a la salud, sino la promoción de la salud y prevención de la enfermedad, tal y como marca el artículo 43 de la Constitución Española.

La disyuntiva en torno a la gestión del gasto sanitario no es una cuestión menor y en algunos países, la prestación farmacológica puede llegar a ser considerada un recurso de primera necesidad. Un informe de la OCDE (18) destaca que:

Las probabilidades de que los hogares con bajos ingresos reporten que no cubren sus necesidades de atención médica y dental por motivos financieros o de otra índole son de cuatro a seis veces más altas que las de los hogares con altos ingresos. En algunos países, como Grecia, el porcentaje de la población que reporta algunas necesidades médicas no cubiertas aumentó en más del doble durante la crisis económica (18 p.2).

En el caso español, se puede decir que, aunque la redistribución del gasto sanitario es superior a la registrada en la década de 1990, esta sigue siendo menor a la época anterior a la crisis económica y financiera (19). De esta manera, el Estado de bienestar se haya en la encrucijada de encontrar fórmulas que promuevan el progreso en relación con la atención a la salud sin que suponga una privación material el acceso a productos farmacéuticos.

La provisión de los medicamentos no es sólo una cuestión que atañe a la esfera privada, desde un paradigma universal, sino también a la pública ya que en determinados territorios la falta de asistencia médica - por ejemplo, a través de las vacunas- puede ser un elemento de riesgo para toda la población. De nuevo emergen las conexiones entre pobreza y salud, así como la privación en el acceso de medicamentos por su precio.

Si tanto los países pobres como la población que se encuentra en situación de pobreza en los países más desarrollados no pueden acceder a productos farmacéuticos, la protección a la salud queda mermada para amplios grupos sociales, restringiéndose el ejercicio de su derecho universal a la salud y generándose una polarización social.

En España, el gasto de la ciudadanía en productos farmacéuticos no ha dejado de incrementarse desde 2012, año de entrada en vigor de dos importantes normas que modificaron la protección social en este país: el Real Decreto-Ley 16/2012 y el Real Decreto-Ley 20/2012. Sin embargo, es necesario conocer sobre quién recae ese mayor esfuerzo económico y si existen personas que tienen más probabilidades de encontrarse en una situación de privación farmacéutica. 
Existen numerosas investigaciones que relacionan la pobreza con el uso de medicamentos. Uno de los estudios más relevantes fue el realizado por López (20) donde evidencia las interconexiones entre pobreza y el acceso a la salud, así como a los productos farmacéuticos, destacando que la primera es un determinante mucho mayor que la segunda y que existe la percepción -por gran parte de la población española- de que "los recursos totales que España gasta en salud no están en consonancia con nuestro nivel de desarrollo" (20 p.25).

Recientes estudios latinoamericanos relacionan la privación material en el acceso a la atención farmacéutica con el sexo, edad, ingresos económicos y residencia. El estudio de Silva et al. (21), pone en evidencia cómo el Programa de Farmacia Popular de Brasil es utilizado en su mayoría por mujeres, entre los 61 y 70 años, cuyo estado civil es casada, no alfabetizada, con ingresos económicos de uno a dos salarios mínimos y residentes en inmuebles alquilados. Los resultados de esta investigación también mostraron que más del 50\% de los encuestados tenían dificultades económicas para adquirir medicamentos en sus procesos patológicos. Por tanto, la disposición hacia el acceso de fármacos depende de la capacidad económica individual y puede ser una forma de privación más, como la alimentaria o energética.

La gestión del derecho de la salud y la democratización del acceso a los productos farmacéuticos deben ser elementos centrales en el análisis y la planificación de las políticas públicas. Además, son objeto de análisis en el actual marco económico de superposición de las desigualdades dentro de un contexto de desarrollo neocapitalista donde los Estados están perdiendo su influencia tanto intra como extra nacional.

\section{Implicaciones de los cambios legislativos en materia farmacéutica: justificación y metodología}

En España el sistema de Seguridad Social y el Sanitario están totalmente diferenciados. A pesar de que los ciudadanos se identifican dentro de las instituciones sanitarias por su número de Seguridad Social, este sistema únicamente se encarga de recaudar ingresos a través de las cotizaciones sociales de trabajadores y empresas para pagar las pensiones y prestaciones por desempleo.

La prestación de medicamentos dentro de los centros sanitarios es gratuita pero no aquellos destinados al consumo domiciliario. Sobre estos medicamentos los ciudadanos españoles siempre han tenido que participar en su financiación a través de un sistema de copago. Sin embargo, la recaudación económica no era el motor que guiaba el establecimiento del copago farmacéutico. Tampoco se pretendía con ello desincentivar a los ciudadanos en el acceso a los mismos. El copago farmacéutico es un sistema orientado a involucrar a los ciudadanos en el costo social de medicamentos. Por tanto, no se trata de un sistema de subsidio al mercado sino de un sistema de cofinanciación. A pesar de ello, siempre han existido colectivos exentos de la participación en el copago, como por ejemplo las personas pensionistas, independientemente de su renta y patrimonio. 
En los últimos años ha existido una extensa producción teórica en torno a las consecuencias del cambio de la regulación en materia de acceso a los medicamentos de la población española. Sin embargo, al ser un fenómeno nuevo en el modelo de bienestar social de este país resulta interesante abordar las implicaciones que tienen las reformas legislativas en la ciudadanía.

Se ha partido de la hipótesis de que los cambios legislativos en materia de productos farmacéuticos, en un contexto de crisis económica con alto desempleo, está haciendo proliferar un nuevo tipo de privación material ligada al acceso a los medicamentos. De confirmarse ésta, tendría un difícil encaje dentro del modelo español de bienestar social en protección a la salud, caracterizado por la atención universal. Además, se podría estar produciendo un proceso de polarización social en el acceso a un bien de primera necesidad en virtud de la capacidad económica de los pacientes.

El objetivo es conocer cuáles son las implicaciones de las modificaciones normativas en el acceso de medicamentos en la población española, así como comprobar si existe un perfil determinado de personas que no pueden acceder a los fármacos. Para ello, se analizará la evolución del gasto público y el gasto privado personal en productos farmacéuticos, la carga que supone para los hogares adquirir estos productos (teniendo en cuenta sexo, edad, nacionalidad, tipo y renta del hogar), así como el número de personas que no han podido acceder a los medicamentos. Se ha empleado una metodología mixta combinando una vertiente cualitativa en la que se han estudiado las consecuencias de las últimas reformas legislativas en España en materia de medicamentos, con una aproximación a las fuentes secundarias más importantes que están abordando este fenómeno: Barómetro Sanitario, Encuesta de Condiciones de Vida y los Informes sobre el Gasto Farmacéutico de la EAE Business School. El Barómetro Sanitario es un estudio de opinión realizado por el Ministerio de Sanidad en colaboración con el Centro de Investigaciones Sociológicas para conocer la opinión de las personas mayores de 18 años sobre la política sanitaria (Ministerio de Sanidad, Consumo y Bienestar Social, 2018). La Encuesta de Condiciones de Vida permite comparar la distribución de ingreso y la exclusión social en un contexto europeo (24). Por último, los Informes sobre el Gasto Farmacéutico en España son estudios anuales que analizan la evolución del gasto farmacéutico tanto a nivel público como de las familias (25).

\section{Análisis de los cambios legislativos en la prestación farmacéutica en España}

La protección farmacéutica está enmarcada dentro de la intervención social del Estado en la regulación del bienestar social, donde cada país desarrolla su propio sistema de gestión siguiendo las características de su modelo de protección social. En España, la Seguridad Social se ha encargado de proveer el acceso de los medicamentos de una forma extensiva a la población, protegiendo especialmente a las personas pensionistas y aquéllas con algún tipo de discapacidad. Otros países establecen similares sistemas de protección ligados a la cobertura sanitaria o seguros privados. 
Las últimas reformas sanitarias llevadas a cabo en España han aumentado los debates actuales en torno al copago farmacéutico. Esta fórmula se instauró en 1967 y durante este tiempo ha existido consenso y estabilidad en torno al mismo. A lo largo de este período se han introducido numerosas reformas legislativas, pero ninguna ha causado la alarma social y el desacuerdo en la población como las implementadas en los años de la crisis económica cuando se aprobaron el Real Decreto-ley 16/2012, de medidas urgentes para garantizar la sostenibilidad del Sistema Nacional de Salud y mejorar la calidad y seguridad de sus prestaciones, la Ley 28/2014, por la que se modifican la Ley 37/1992 del Impuesto sobre el Valor Añadido y el Real Decreto Legislativo 1/2015. Estas normas introdujeron cambios sustanciales en el acceso a la salud y a las prestaciones farmacéuticas. Sin embargo, el alcance en la población ha sido mucho mayor ya que han venido acompañadas de otras normas jurídicas dirigidas a la restricción del gasto y limitación de derechos en el conjunto de espacios sociales, especialmente los recogidos en el Real Decreto-ley 20/2012.

En los últimos años han calado en el imaginario colectivo las dificultades en el acceso de la población a la prestación farmacéutica por los altos niveles de copago establecidos. Sin embargo, no se cuestiona tanto el sistema de copago en su totalidad, sino la intensidad del mismo y el cambio radical que implicó. La mayoría de las normas jurídicas relativas al copago farmacéutico se han mantenido vigentes desde el año 1980, donde se establecía un copago general situado en el $40 \%$. En la última década se han implantado grandes reformas que han modificado sustancialmente la protección social de los medicamentos en España, por ejemplo, en el año 2006 se excluyeron del catálogo farmacéutico un gran número de medicamentos estéticos, publicitarios y homeopáticos. Sin embargo, la norma jurídica que ha tenido una mayor repercusión ha sido el Real Decreto-Ley 16/2012. Los cambios más importantes fueron el incremento del copago farmacéutico y su vinculación a la renta anual, el establecimiento de copago para los pensionistas y una cobertura especial para las personas en vulnerabilidad social (perceptores de rentas de subsistencia, de pensiones no contributivas y subsidios por desempleo). Estos cambios se pueden observar la tabla 1. 
Gema Martínez-Gayo / José Ángel Martínez-López

Tabla 1 Cambios legislativos más relevantes en relación al copago farmacéutico en España

\begin{tabular}{|c|c|}
\hline Norma Juridica & Modificaciones legales más relevantes \\
\hline Decreto $3157 / 1966$ & $\begin{array}{l}\text { Se establece un copago mínimo del } 16,6 \% \text { de medicamento } \\
\text { con un limite de } 50 \text { pesetas }{ }^{/ 1} \text {. }\end{array}$ \\
\hline Real Decreto $945 / 1978$ & $\begin{array}{l}\text { Se establece un copago general del } 30 \% \text { sin límite } \\
\text { establecido. }\end{array}$ \\
\hline $\begin{array}{l}\text { Real Decreto } \\
1605 / 1980\end{array}$ & Aumento del copago general al $40 \%$ sin límite establecido. \\
\hline Ley $29 / 2006$ & $\begin{array}{l}\text { Se excluyen del catálogo de medicamentos los productos } \\
\text { estéticos, publicitarios, homeopáticos, etc., y se establece una } \\
\text { aportación normal ( } 40 \% \text { sin límite establecido) y otra } \\
\text { reducida ( }(10 \% \text { con un máximo de } 2,64 €) \text {. }\end{array}$ \\
\hline $\begin{array}{l}\text { Real Decreto-Ley } \\
16 / 2012\end{array}$ & $\begin{array}{l}\text { El copago se determina en virtud de la capacidad económica } \\
\text { estableciéndose solo } 3 \text { intervalos: A) Aporte del } 60 \% \text { cuando } \\
\text { los ingresos sean iguales o superiores a } 100.000 € \text {. B) Aporte } \\
\text { del } 50 \% \text { cuando los ingresos sean de entre } 18.000-100.00 € \text {. } \\
\text { C) Aporte del } 40 \% \text { cuando sus ingresos sean inferiores a } \\
18.000 € \text {. La aportación de los pensionistas es del } 10 \% \text { aunque } \\
\text { se establecen excepciones y límites máximos sean sus } \\
\text { ingresos económicos. } \\
\text { También se recogen excepciones y una cobertura diferenciada } \\
\text { para perceptores de una renta de integración social, } \\
\text { beneficiarios de pensiones no contributivas }{ }^{2} \text { o subsidios por } \\
\text { desempleo. Sin embargo, no se recoge ninguna protección } \\
\text { diferenciada para las personas perceptoras de una prestación } \\
\text { por desempleo }{ }^{2} \text { o quienes carezcan de ingreso alguno. }\end{array}$ \\
\hline
\end{tabular}

Fuente: BOE, elaboración propia.

La situación actual pone en evidencia la falta de equidad al establecer los estratos en relación al copago configurando únicamente tres niveles. Un ejemplo de ello es que participan en el copago por igual quienes tienen unos ingresos anuales de $18.001 €$ que quienes tienen una renta de hasta $100.000 €$. Otro elemento destacado es que los pensionistas vuelven a pagar por acceder a los medicamentos; con lo cual se retroceden décadas respecto al copago de medicamentos. Finalmente, destaca la falta de protección social para las personas desempleadas perceptores de la prestación por desempleo ${ }^{1}$ y que aquéllos que carecen de ingreso alguno. Estudios como el realizado por Rodríguez-Feijoó et al. (22), muestran cómo estas medidas:

[han afectado] principalmente los activos con rentas del hogar inferiores a 900 euros mensuales y los jubilados con rentas inferiores a 600 euros, y adicionalmente existen diferencias por razón de la situación laboral (22 p.6).

De forma complementaria, se aprobó la Resolución del 2 de agosto de 2012, de la Dirección General de Cartera Básica de Servicios del Sistema Nacional de Salud y Farmacia, por la que se procede a la actualización de la lista de medicamentos que quedan excluidos de la prestación farmacéutica en el Sistema Nacional de Salud. A través de esta Resolución se 
dejó de subvencionar más de 400 productos farmacéuticos de uso cotidiano como antiácidos, antidiarreicos, pomadas para aliviar dolores, los destinados a cubrir algunos tipos de afecciones inflamatorias o problemas alérgicos. Por tanto, se produce una doble limitación en la cobertura sanitaria al incrementar el sistema de copago y excluir simultáneamente un gran número de productos farmacéuticos de la cobertura sanitaria pública. Como afirman Martínez-López et al. (23):

Lo que para unos supone una medida para mejorar la eficiencia del gasto público y hacerlo sostenible, es para otros una manera de debilitar las prestaciones del Estado de bienestar, que acaba por empeorar la situación de quienes son más vulnerables y no pueden acceder, o lo hacen con dificultad, a los fármacos prescritos (23 p.13).

A modo de síntesis, uno de los aspectos más destacados de estas reformas legislativas es que cargan parte de la financiación sobre los pensionistas -quienes que ya participaron en la subvención del sistema en su edad laboral- y obvia a los perceptores de prestaciones por desempleo como colectivo necesitado de una mayor protección social. Para las personas jubiladas con escasos ingresos y los desempleados sin acceso a prestaciones sociales puede constituir un nuevo tipo de privación material: la privación farmacéutica.

\section{A vueltas entre la protección y privación de medicamentos en España}

España se encuentra en una posición elevada entre los países que hacen parte de la OCDE, en cuanto a la aportación del porcentaje del Producto Interior Bruto en medicamentos, material y otros productos farmacéuticos con un 1,63\% del mismo. Superando esta cifra están Grecia, Hungría y Estados Unidos todos ellos sobrepasando el 2\% del PIB, y también Eslovaquia y Canadá, con cifras muy próximas. En cuanto al gasto público, en esta partida también se encuentra en la zona alta; aunque la tendencia en los últimos años es a reducirse, especialmente desde 2009 donde se alcanzó el 1,29\% del PIB. Por su parte, el gasto privado ocupa un puesto intermedio en la clasificación internacional, aunque tiende a incrementarse. Por último, añadir que entre 2009 y 2014 se ha reducido el gasto total, en estos productos, en un $0,77 \%$, siendo más acusado para el último año donde la merma superó el 3,4\% (25).

Entre 2010 y 2013 el gasto farmacéutico público español sufrió un continuado y acusado descenso. Esta tendencia se atenuó en los dos últimos años produciéndose un leve incremento, el cual no es suficiente para compensar el descenso de prácticamente un $15 \%$ entre 2007 y 2015. La evolución del gasto público por receta, en ese mismo período, es similar, lo que tuvo una incidencia en el esfuerzo personal de los ciudadanos para acceder a los fármacos. De tal forma que, entre 2007 y 2015, el gasto farmacéutico privado por persona se incrementó en casi un $16 \%$. (ver figura 1 ). 


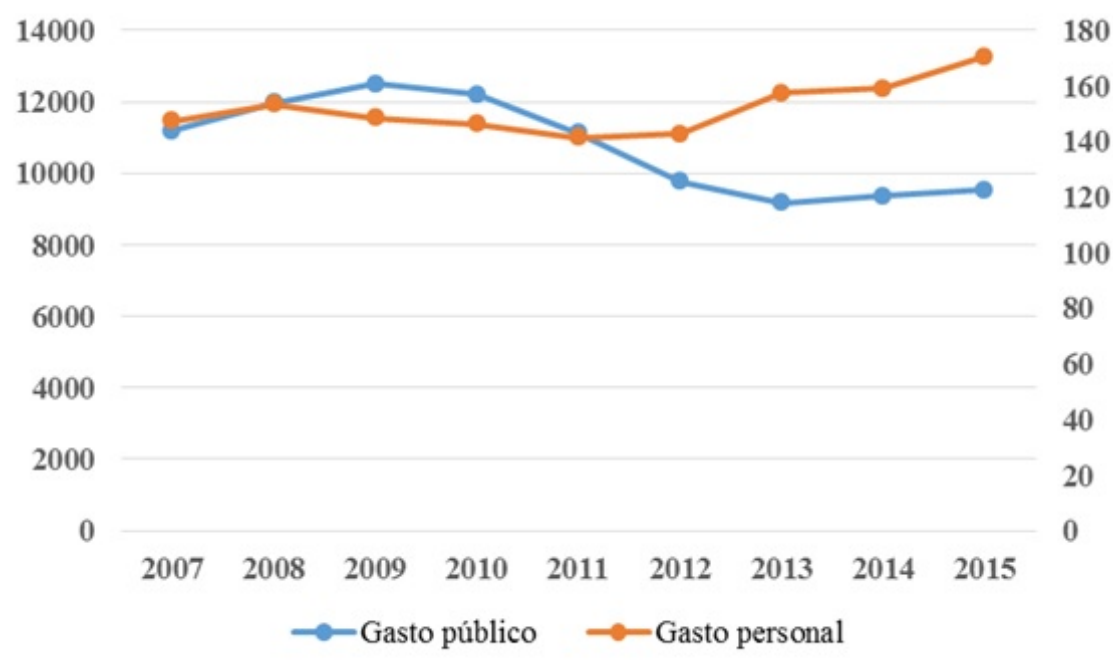

Figura 1 Evolución del gasto farmacéutico público total (millones de euros), del gasto farmacéutico personal privado (euros) y variación (\%) Fuente: elaboración propia con datos de EAE Business School (25).

Las mujeres, con independencia de la edad analizada, presentan mayor gasto en productos farmacéuticos; especialmente a partir del año 2013 cuando empiezan a notarse los efectos de la entrada en vigor de la Reforma Sanitaria. También la edad de las personas influye en el montante de gasto en medicamentos, siendo las que se encuentran en edad de jubilación las que más gastan en este tipo de productos. Los hombres que superan los 65 años son los que han visto en mayor medida incrementar su gasto farmacéutico en estos ocho años, prácticamente en un 39\%. Además, aquellos hogares formados por una persona sola que supera la citada edad, son los que más gastan y en los que más se ha incrementado el monto del gasto farmacéutico llegando a superar, en 2015, los 365 euros (24). Esta situación también ha repercutido en la dificultad de acceso a los medicamentos (ver tabla 2 ). 
Tabla 2 Hogares (porcentajes horizontales) según carga de los gastos en medicamentos según sexo y edad, nacionalidad, tipo de hogar y quintil de renta por unidad de consumo

\begin{tabular}{|l|c|c|c|}
\hline & Carga pesada & Carga razonable & $\begin{array}{c}\text { Sin carga / } \\
\text { No consume }\end{array}$ \\
\hline Edad y Sexo & \multicolumn{3}{|l|}{} \\
\hline H 45 a 64 años & 8,3 & 44,2 & 47,4 \\
\hline H 65 a más años & 7,2 & 51,0 & 41,8 \\
\hline M 45 a 64 años & 9,0 & 44,6 & 46,3 \\
\hline M 65 a más años & 10,4 & 50,9 & 38,6 \\
\hline Nacionalidad & \multicolumn{5}{|l|}{} \\
\hline Española & 46,2 & 46,1 \\
\hline Extranjera (UE) & 7,1 & 48,6 & 44,3 \\
\hline Extranjera (Resto) & 10,6 & 44,0 & 45,4 \\
\hline Tipo de hogar & \multicolumn{5}{|l|}{} \\
\hline H solo < 65 años & 7,8 & 36,8 & 55,4 \\
\hline M sola < 65 años & 9,0 & 40,4 & 50,6 \\
\hline M sola 65 o +años & 10,3 & 52,1 & 37,7 \\
\hline $\begin{array}{l}\text { Adulto solo hijos } \\
\text { dependientes }\end{array}$ & 8,8 & 38,7 & 52,4 \\
\hline $\begin{array}{l}\text { 2 adultos, al menos } \\
\text { uno 65 o más años }\end{array}$ & 8,8 & 51,3 & 39,9 \\
\hline Quintil de renta por unidad de consumo & & 46,9 \\
\hline Primer quintil & 11,9 & 42,8 & 45,2 \\
\hline Segundo quintil & 10,0 & 45,9 & 44,0 \\
\hline Tercer quintil & 7,5 & 46,7 & 45,8 \\
\hline Cuarto quintil & 5,2 & 47,9 & 46,9 \\
\hline Quinto quintil & 4,6 & 47,0 & \\
\hline
\end{tabular}

Fuente: elaboración propia con datos del INE (24).

Por franjas etarias a las mujeres siempre les supone una carga más pesada el acceso a los medicamentos que a los hombres de su misma edad, ya que son las mujeres de entre 45 y 64 años y las de 65 o más edad las más afectadas. En este último caso, mujeres en edad de jubilación, supera el 10\% hogares los que supone una carga pesada el acceso a estos bienes. En cuanto a la nacionalidad la mayor carga o dificultad corresponde con aquellos hogares de personas extranjeras, de fuera de la UE, con prácticamente un 11\%, seguidos de las españolas.

En el caso de los tipos de hogares, existen tres variables explicativas a la hora de encontrar pesada la carga del gasto en medicamentos: ser mujer sola en el hogar, el tener hijos dependientes y superar los 65 años. En el primer caso, mujer sola, tanto menor de 65 años como mayor, siendo en este último caso las más afectadas superando el 10\% de estos hogares. Respecto a los hijos dependientes afecta especialmente el hecho de que solo haya un adulto en el hogar y, por último, aquellos hogares en el que al menos uno de los adultos supere los 65 años.

La última variable analizada, que es el quintil de renta por unidad de consumo, nos deja datos donde los dos primeros quintiles, que representan al 40\% con menor renta, son los que más dificultades presentan para acceder a los medicamentos, con un $12 \%$ y un $10 \%$ respectivamente. Mientras que para el $20 \%$ de los hogares más ricos menos de un $5 \%$ le supondría una carga pesada. Hay más de siete puntos porcentuales de diferencia entre el primer y quinto quintil de 
renta. A la hora de poder afrontar los gastos estas dificultades han contribuido a que una parte de la ciudadanía no pueda acceder a la medicación prescrita por su facultativo (ver tabla 3).

Tabla 3 Personas que han dejado de tomar los medicamentos prescritos en la sanidad pública por no poder permitírselo y variación $(\%)$

\begin{tabular}{|c|c|c|c|c|c|c|c|}
\hline & 2013 & 2014 & 2015 & 2016 & 2017 & 2016-17 & 2013-17 \\
\hline $\begin{array}{l}\text { No pueden } \\
\text { permitírselo }\end{array}$ & 5,2 & 4,5 & 4,0 & 4,4 & 4,7 & $+0,3$ & $-0,5$ \\
\hline $\begin{array}{l}\text { Si pueden } \\
\text { permitírselo }\end{array}$ & 92,66 & 94,8 & 95,5 & 89,5 & 88,6 & & \\
\hline $\begin{array}{l}\text { No se los han } \\
\text { recetado último año }\end{array}$ & - & -- & - & 5,7 & 6,6 & & \\
\hline N.C. & 2,15 & 0,7 & 0,5 & 0,4 & 0,1 & & \\
\hline$(\mathrm{N})$ & (7750) & (7721) & (7746) & (7752) & (7735) & & \\
\hline
\end{tabular}

Fuente: elaboración propia según los datos del Barómetro Sanitario de varios años.

Un año después de la entrada en vigor de la llamada Reforma Sanitaria se registra un porcentaje de personas que no puede acceder a los medicamentos prescritos de algo más del $5 \%$, dato que se verá reducido en los dos años posteriores. En los años con los que se cierra el análisis, 2016 y 2017, vuelve a incrementarse este porcentaje cerrando el período con un dato cercano al 5\% inicial (26).

Si además del acceso a los medicamentos tenemos en cuenta la imposibilidad de seguir tratamientos médicos o dietas, en 2013 un 16\% de las personas no habrían podido acceder a estos por problemas económicos. Este dato es notablemente superior al registrado en 2007, ya que esta nueva cifra prácticamente lo triplica. También se duplica el porcentaje de personas que ha pasado o sufre hambre en la actualidad, y en los hogares en situación de exclusión social duplica el número de personas que ha dejado de recibir atención médica por hallarse en lista de espera a la de los hogares que se hayan integrados (19).

\section{Conclusiones}

El Estado de bienestar construido en España en la década de 1980, con el impulso de la sanidad universal, muestra grietas en su desarrollo actual. Los avances en derechos sociales han estado íntimamente vinculados con los ciclos económicos. Esta situación es palpable en la crisis económica iniciada en 2008 donde gran parte de las actuaciones gubernamentales se han centrado en la restricción del gasto en políticas sociales. En los últimos años en España se ha llevado a cabo una reducción del gasto farmacéutico público, a la par, que el gasto personal privado se ha incrementado aún en mayor medida. A pesar de ello, el derecho a la salud ha sido uno de los más firmes dentro del modelo de bienestar social español aglutinando tanto la atención primaria, especializada, hospitalaria como a través de la cobertura de los medicamentos. 
Los productos farmacéuticos constituyen un bien de primera necesidad y un elemento indispensable para gozar de buena salud. Como consecuencia de los ajustes presupuestarios exigidos para equilibrar la finanzas nacionales, se han incorporado cambios legales sustanciales poniendo en evidencia dos aspectos: a) la debilidad de nuestro modelo de bienestar social que vincula el avance en derechos sociales la economía y, b) las reformas legislativas en el sistema de copago se han establecido con una escasa progresividad, afectando con mayor intensidad a las personas más vulnerables.

Existe una tendencia global en gran parte de los países desarrollados a reducir el papel del Estado como regulador social y a transferir la responsabilidad del bienestar social hacia el ámbito individual. Ello genera procesos de polarización social y agrava las diferencias entre ciudadanos ya que no puede decirse que la mayoría de los hogares en la actualidad puedan cubrir sus necesidades básicas únicamente a través del empleo. La privación material en España en el ámbito de los productos farmacéuticos, que reflejan diferentes fuentes de datos, marca un hito en la atención a la salud y cuestiona el modelo implantado hasta ahora. No se puede gozar de unos niveles aceptables de salud si la ciudadanía no puede acceder a medicamentos con los cuales hacer frente a sus patologías. La intensidad de este fenómeno es una novedad dentro de la política sanitaria.

Los colectivos que se están viendo más afectados por la carga económica de los medicamentos son las mujeres (21), las personas de nacionalidad extranjera no perteneciente a la UE, los hogares monoparentales con hijos dependientes, los que superan la edad de jubilación y los dos primeros quintiles de renta (26). Este hecho no encaja con la conceptualización de que los medicamentos deben tener un precio asequible para corresponder con los principios de equidad que favorezcan el bienestar de la población (23), ya que prácticamente el 5\% de la población en 2017 no podía acceder a los productos farmacéuticos prescritos. Por tanto, la privación de acceso a los medicamentos es un nuevo tipo de carencia material que debe ser estudiada por los indicadores tradicionales que miden la pobreza y desigualdad social.

Se ha podido comprobar nuestra hipótesis de investigación dado que las reformas legislativas en materia de copago sanitario están produciendo dificultades en el acceso a estos bienes a las personas que se encuentran en una peor situación socioeconómica. A su vez se agudizan los procesos de polarización social con relación al derecho a la salud.

Nuestra investigación presenta nuevas líneas de análisis que resultarían interesantes de desarrollar en los próximos años. Entre ellas se destaca la posibilidad de conocer cómo siguen afectando las reformas legislativas en los medicamentos a la población a largo plazo o si tras la recuperación económica se mantienen los niveles de privación material en los mismos perfiles. Nos hallamos ante un fenómeno complejo que requiere de un seguimiento en el futuro. 
Gema Martínez-Gayo / José Ángel Martínez-López

\section{Referencias}

1. Marshall TH. Citizenship and social class. Cambridge: The syndics of the Cambridge University Press; 1950.

2. Iriart C, Merhy EE. Inter-capitalistic disputes, biomedicalization and hegemonic medical model. Interface (Botucatu). 2017;21(63): 1005-1016. https://doi.org/10.1590/1807-57622016.0808

3. Nichter M. Pharmaceuticals, the commodification of health, and the health care-medicine use transition. En: Nichter M, Nichter M (eds.) Anthropology and International Health. London: Routledge; 2003. p.268-333.

4. Páez, R. La investigación internacional de nuevos medicamentos: una valoración desde la justicia global. Revista Latinoamericana de Bioética. 2016;16(31-2): 188-213. https://doi.org/10.18359/rlbi.1689

5. Díaz-Mosquera SP, Rodríguez-Villamil LN, Valencia-González AM. Análisis de publicaciones en promoción de la salud: una mirada a las tendencias relacionadas con prevención de la enfermedad. Rev Gerenc Polít Salud. 2015; 14(28): 32-47. https://doi.org/10.11144/Javeriana.rgyps18-28.apps

6. Rawls J. Teoría de la justicia. Cambridge: The Belknap Press of Harvard University Press; 1971.

7. Sen A. Nuevo examen de la desigualdad. Madrid: Alianza Editorial; 2003.

8. Vargas-Chaves I. Redimensión de las políticas públicas frente al acceso a medicamentos: entre la ausencia, la permisividad y el abandono estatal. Estudios Socio-Jurídicos. 2015;17(1):169-193. https://doi.or $\mathrm{g} / 10.12804 / \mathrm{esj} 17.01 .2014 .05$

9. Cantero J. Constitución y derecho a la protección de la salud. En: Francisco Balaguer F, Arana E (eds.) Libro homenaje al profesor Rafael Barranco Vela. Cizur Menor, Navarra: Thomson Reuters-Civitas; 2014. p.1511-1555.

10. Jiménez-Martín S, Viola AA. El sistema de salud en España en perspectiva comparada. Fedea; 2014. http://sanidad.fedea.net/docs/informe.pdf.

11. Jiménez-Martín S, Viola AA. Consumo de medicamentos y copago farmacéutico. Fedea; 2016. http:// documentos.fedea.net/pubs/eee/eee2016-06.pdf

12. Monteverde M, Tomas S, Acosta L, Garay S. Envejecimiento poblacional y magnitud de la dependencia en Argentina y México: perspectiva comparada con España. ALAP Revista Latinoamericana de Población. 2016;10(18): 135-154. https://doi.org/10.31406/relap2016.v10.i1.n18.6

13. Martínez-López JA, Frutos L, Solano JC. Los usos de las prestaciones económicas de la dependencia en el municipio de Murcia. Un estudio de caso. Revista Española de Sociología. 2017;26(3): 97-113. https://doi.org/10.22325/fes/res.2017.37

14. Guttier MC, Pinto M, Luiza L, Bertoldi AD. Impact of interventions to promote the use of generic drugs: a systematic review. Ciênc Saúde Colet. 2017;22(8): 2627-2644. https://doi.org/10.1590/1413-8123 2017228.05762017 
15. Institute for Human Data Science. The global use of medicine in 2019 and Outlook to 2023 [Internet]. IQVIA; 2019 [citado el 22 de abril de 2020]. 60 p. Recuperado de https://informatori.it/wp-content/ uploads/2019/03/the-global-use-of-medicine-in-2019-and-outlook-to-2023.pdf.

16. OCDE. Health at a glance 2017. OCDE; 2017. https://www.oecdilibrary.org/docserver/4dd50c09en.pdf ?expires $=1587122407 \& \mathrm{id}=\mathrm{id} \&$ accname $=$ guest $\&$ checksum=41D3AA2CF271F0A196A0CB7ED1B 21347

17. Klein E, Van Boeckel, TP, Martínez, EM, Pant E, Granda S, Levin SA, Goossens H. Global increase and geographic convergence in antibiotic consumption between 2000 and 2015. PNAS. 2018;115(15): 3463-3470. https://doi.org/10.1073/pnas.1717295115

18. OCDE. Health at a Glance 2015. OCDE; 2015. http://www.oecd.org/health/health-systems/health-at-a -glance-19991312.htm.

19. Foessa. VII Informe sobre exclusión y desarrollo social en España. Fundación Foessa; 2014. 686 p. htt ps://www.foessa2014.es/informe/uploaded/descargas/VII_INFORME.pdf

20. López I Casasnovas G. Una valoración de opciones para la política sanitaria española en un contexto descentralizado. Cuadernos de Ciencias Económicas y Empresariales. 2005;49: 19-32. https://dialn et.unirioja.es/servlet/articulo?codigo $=2251127$

21. Silva E, Paiva A, Bitu N, Peres Do Souto R, Rocha MDF, et al. Perfil socioeconómico e farmacoterpêutico dos usuarios do programa farmacia popular na unidade de Juazeiro do Norte-CE. Infarma. 2017;29(3): 255-263. http://dx.doi.org/10.14450/2318-9312.v29.e3.a2017.pp255-263

22. Rodríguez-Feijoó S, Rodríguez-Caro A. El copago farmacéutico en España tras la reforma del año 2012 desde la perspectiva del usuario. ¿Evidencias de inequidad? Gac Sanit. 2019;1806: 1-7. https://doi. org/10.1016/j.gaceta.2019.09.009

23. Martínez-López JA, Martínez-Gayo G. Implicaciones del aumento del copago farmacéutico en España: una nueva privación material. Convergencia: Revista de Ciencias Sociales. 2019;81: 1-21. https://d oi.org/10.29101/crcs.v26i81.10327

24. Instituto Nacional de Estadística. Encuesta de Condiciones de vida. Módulo de Salud del año 2017. Instituto Nacional de Estadística; 2017 https://www.ine.es/dynt3/inebase/index.htm?type=pcaxis\&p ath $=/ \mathrm{t} 25 / \mathrm{p} 453 /$ modulo $/ 2017 / \&$ file $=$ pcaxis $\& \mathrm{~L}=0 \& \mathrm{dh}=0 \&$ capsel $=0$.

25. EAE Business School. El gasto farmacéutico en España 2016. EAE Business School; 2016. https://www.eae.es/actualidad/faculty-research/el-gasto-farmaceutico-publico-por-habitante-s ube-por-segundo-ano-consecutivo-y-se-situa-en-20531eu.

26. Ministerio De Sanidad, Consumo y Bienestar Social. Barómetro Sanitario; 2018. https://www.mscbs.g ob.es/estadEstudios/estadisticas/ BarometroSanitario/home_BS.htm

Notas

* Artículo de investigación.

1 A diferencia del subsidio por desempleo tiene un carácter contributivo. 